\title{
Intensification des transformations du lactose dans la fabrication du Cheddar
}

\author{
par \\ GOODA* (E.), BEDNARSKI* (W.), KUNCEWICZ (A.), \\ POZNANSKI (S.) et KOWALEWSKA (Jadwiga)
}

\section{INTRODUCTION}

En technologie fromagère, les transformations enzymatiques du lactose commencent déjà au cours du développement des bactéries dans le lait préparé en vue de la fabrication. L'intensité et la direction des transformations du lactose dans le lait et dans les fromages dépendent du type de tromage produit et des facteurs technologiques. C'est aussi le nombre et la qualité des microbes des levains, avec leur activité $\beta$-galactosidase intracellulaire, $[1,5,12]$, qui déterminent le degré d'hydrolyse du lactose et la durée de chaque étape de la fabrication du fromage. On peut intensifier l'hydrolyse du lactose, dans le lait et dans le fromage, en ajoutant une préparation de $\beta$-galactosidase $[1,5,9,15]$. Le degré d'hydrolyse du lactose (teneurs en glucose et en galactose) peut être déterminé par différentes méthodes $[4,11,14,18,19]$. Les plus répandues sont celles qui mettent à profit les propriétés reproductrices des sucres [13, 19]. On connaît aussi les méthodes enzymatiques [6], la chromatographie sur papier [4, 14], la chromatographie d'échange d'ions [17].

Dans ce travail la chromatographie en phase gazeuse a été mise en œuvre pour déterminer les teneurs en sucres dans le fromage. Le but du travail présenté est de préciser l'intérêt de l'utilisation de $\beta$-galactosidase dans la fabrication du Cheddar par détermination de la cinétique des transformations du lactose et examen des possibilités de raccourcir le "process " technologique, surtout la période de maturation du fromage.

* Institut du Génie et de la Biotechnologie Alimentaire, Académie Agricole Technique à Olsztyn. 


\section{MATERIEL ET METHODES}

\section{a) Fabrication du Cheddar}

Dans 50001 de lait pasteurisé, renfermant 2,8 p. 100 de matière grasse, refroidi à $37^{\circ} \mathrm{C}$ on a ajouté $0,1 \mathrm{p} .100$ de la préparation Maxilact « 40000 » de $\beta$-galactosidase de la levure Kluyveromyces lactis présentant une activité de 33000 unités ONPG/gm (ortho-nitrophénylo $\beta$ - $\gamma$-galactopiranozyde). L'hydrolyse du lactose dans le lait a été effectuée pendant $30 \mathrm{~min}$ avant l'addition de levain. Celui-ci contenait des bactéries appartenant aux espèces Streptococcus lactis, Streptococcus cremaris, Lactobacillus bulgaricus et Lactobacillus helveticus. On a effectué trois fabrications expérimentales des fromages d'après la même technologie.

Parallèlement, on a fabriqué des fromages sans addition de $\beta$-galactosidase.

On a déterminé le rendement de la production de fromage en les pesant et en rapportant la quantité de matière sèche à 10001 de lait.

\section{b) Analyse des fromages}

Les fromages ont été analysés directement après la fabrication et une fois par mois pendant les 5 mois de la maturation. Ont été déterminées : la teneur en matières azotées totales d'après la méthode Kjeldahl, la teneur en matières azotées solubles à $\mathrm{pH} 4,6$ [10], les teneurs en azote peptidique, azote aminé et azote non protéique [7, $17,16]$.

On a effectué également un examen microbiologique des fromages en fin de pressage, au temps 14 jours et après $1,2,3$, 4, et 5 mois de maturation. Les bactéries protéolytiques ont été dénombrées sur le milieu de Frazier [3], les bactéries acidifiantes sur milieu lactosé au bleu de Chine [3] et les germes totaux sur un milieu gélosé préparé avec du lait et du glucose [3].

\section{c) Analyse chromatographique des sucres [2]}

$20 \mathrm{~g}$ de fromage ont été additionnés de $280 \mathrm{ml}$ d'éthanol à 90 p. 100 et la suspension a été homogénéisée par agitation à la vitesse angulaire de $150 \mathrm{rd} / \mathrm{s}$. Ensuite l'échantillon a été centrifugé à $1800 \mathrm{~g}$ pendant $5 \mathrm{~min}$. On récupérait le surnageant, on ajoutait, comme étalon interne, du mésoinositol en quantité répondant à la concentration des sucres dans l'échantillon et ensuite on séchait par sublimation. Les sucres ont été extraits par la pyridine dans un bain d'eau bouillante avec utilisation d'un réfrigérant à reflux. 0,5 ml 
d'extrait a été séché par lyophilisation et au résidu sec on a ajouté $1 \mathrm{ml}$ d'un mélange sililant-SIL Mix HTP 319.

Les conditions de séparation ont été les suivantes :

- chromatographe en phase gazeuse Pye Unicam (série 104) détecteur par ionisation de flammes (FID) ;

- colonne de 2,1 $\mathrm{m}$ remplie de CV-17 à 3 p. 100 sur chromosorb HP 80/100 mesh. L'argon sert de gaz vecteur, la vitesse d'écoulement est de $30 \mathrm{ml} / \mathrm{min}$ et la température de la colonne passe de 145 à $324^{\circ} \mathrm{C}$ avec un $\Delta \mathrm{t}$ de $6^{\circ} \mathrm{C}$ par $\min$;

- température du détecteur $340^{\circ} \mathrm{C}$.

La séparation chromatographique des dérivés sililés constituait la base de l'analyse qualitative et quantitative des sucres libres dans le fromage.

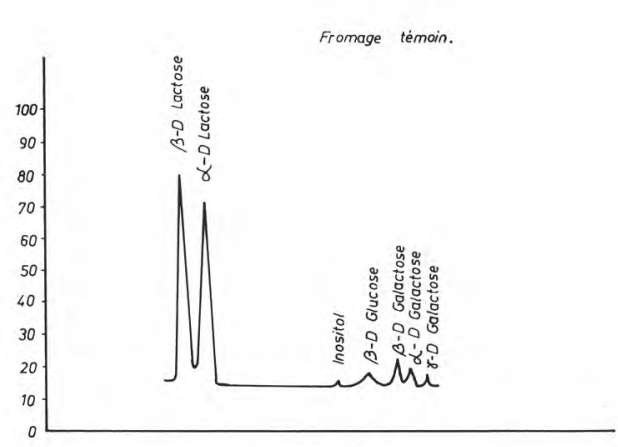

fig. $1 \mathrm{a}$

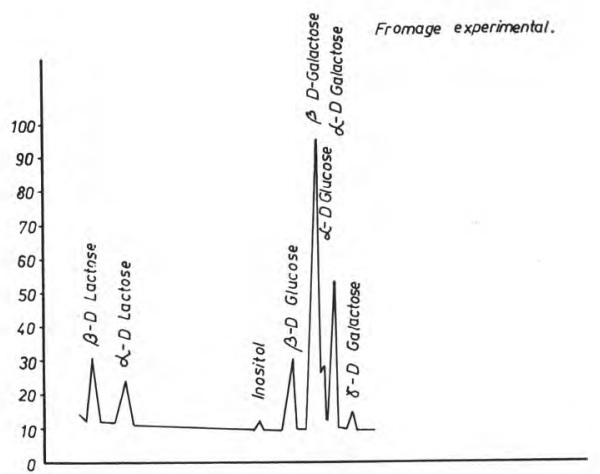

fig. $1 \mathrm{~b}$

Diagrame standard.

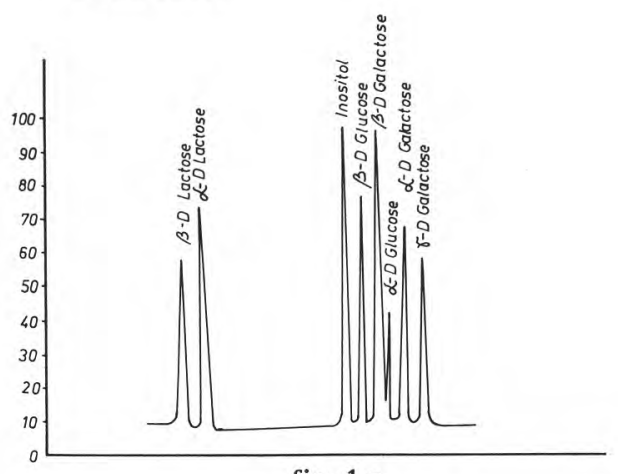

fig. $1 \mathrm{c}$

fig. 1

Séparation chromatographique des sucres dans le fromage $6 \mathrm{~h}$ après la pression. 
TABLEAU 1

Teneurs en sucres dans les fromages ( $\mathrm{mg} / 100 \mathrm{~g}$ de fromage)

\begin{tabular}{|c|c|c|c|c|c|c|c|c|c|c|c|c|c|c|}
\hline \multirow{3}{*}{$\begin{array}{c}\text { Temps } \\
\text { de la } \\
\text { maturation }\end{array}$} & \multicolumn{2}{|c|}{$\alpha+\beta$ lactose } & \multicolumn{2}{|c|}{$\alpha-\Delta$ galactose } & \multicolumn{2}{|c|}{$\beta-\Delta$ galactose } & \multicolumn{2}{|c|}{$\gamma$ galactose } & \multicolumn{2}{|c|}{ Galactose total } & \multicolumn{2}{|c|}{$\beta-\Delta$ glucose } & \multicolumn{2}{|c|}{ Sucres totaux } \\
\hline & A & B & A & B & A & B & A & B & A & B & A & B & A & B \\
\hline & $\mathrm{mg}$ & $\mathrm{mg}$ & $\mathrm{mg}$ & $\mathrm{mg}$ & $\mathrm{mg}$ & $\mathrm{mg}$ & $\mathrm{mg}$ & $\mathrm{mg}$ & $\mathrm{mg}$ & $\mathrm{mg}$ & $\mathrm{mg}$ & $\mathrm{mg}$ & $\mathrm{mg}$ & $\mathrm{mg}$ \\
\hline $\begin{array}{l}6 \mathrm{~h} \text { après } \\
\text { pressage }\end{array}$ & 543,2 & 1792,6 & 416,5 & 119,7 & 1030,3 & 289,3 & 48,5 & 48,8 & 1495,3 & 457,8 & 465,3 & 100,3 & $2557,4^{*}$ & 2350,7 \\
\hline $\begin{array}{l}12 \text { h après } \\
\text { pressage }\end{array}$ & 460,2 & 935,0 & 451,3 & 327,5 & 751,5 & 819,3 & 28,6 & 52,7 & 1231,4 & 1199,5 & 308,2 & 113,3 & $3035,9 * *$ & 2247,8 \\
\hline $2 \mathrm{j}$ & 410,0 & 689,5 & 248,6 & 344,7 & 634,4 & 675,4 & 20,6 & 40,5 & 903,6 & 1060,6 & 207,4 & 117,9 & 1521,0 & 1868,1 \\
\hline $3 \mathrm{j}$ & 377,6 & 656,9 & 217,1 & 344,5 & 503,2 & 651,5 & 20,0 & 32,9 & 740,3 & 1028,9 & 135,8 & 120,3 & 1253,7 & 1806,1 \\
\hline $4 \mathrm{j}$ & 295,7 & 625,2 & 148,5 & 344,8 & 398,8 & 604,2 & 21,3 & 30,7 & 568,6 & 979,7 & 110,4 & 127,4 & 974,7 & 1732,3 \\
\hline $7 \mathrm{j}$ & 212,1 & 459,2 & 101,0 & 369,6 & 324,3 & 453,1 & 13,9 & 36,9 & 444,2 & 859,6 & 102,2 & 98,1 & 758,5 & 1916,9 \\
\hline $15 \mathrm{j}$ & 167,6 & 179,1 & 101,9 & 303,7 & 168,7 & 430,3 & 11,0 & 29,7 & 201,6 & 763,7 & 65,0 & 114,2 & 514,2 & 1057,0 \\
\hline $30 \mathbf{j}$ & 97,5 & 121,4 & 14,1 & 35,7 & 37,4 & 42,9 & 5,9 & 5,9 & 57,4 & 84,5 & 9,1 & 22,8 & 164,0 & 228,7 \\
\hline $60 \mathrm{j}$ & 69,0 & 90,9 & 1,5 & 0,9 & 6,9 & 6,6 & 0,3 & 0,0 & 8,7 & 9,5 & 2,0 & 0,9 & 79,7 & 101,3 \\
\hline \multicolumn{7}{|c|}{$\begin{array}{l}\mathrm{A}=\text { Fromage expérimental } \\
\mathrm{B}=\text { Fromage témoin. }\end{array}$} & \multicolumn{8}{|c|}{$\begin{array}{l}\text { On admet } 53,6 \mathrm{mg} \text { de } \alpha-\Delta \text { glucose / } 100 \mathrm{~g} \text { de fromage ce qui } \\
\text { représente } 2,09 \text { p. } 100 \text { des sucres totaux. }\end{array}$} \\
\hline
\end{tabular}




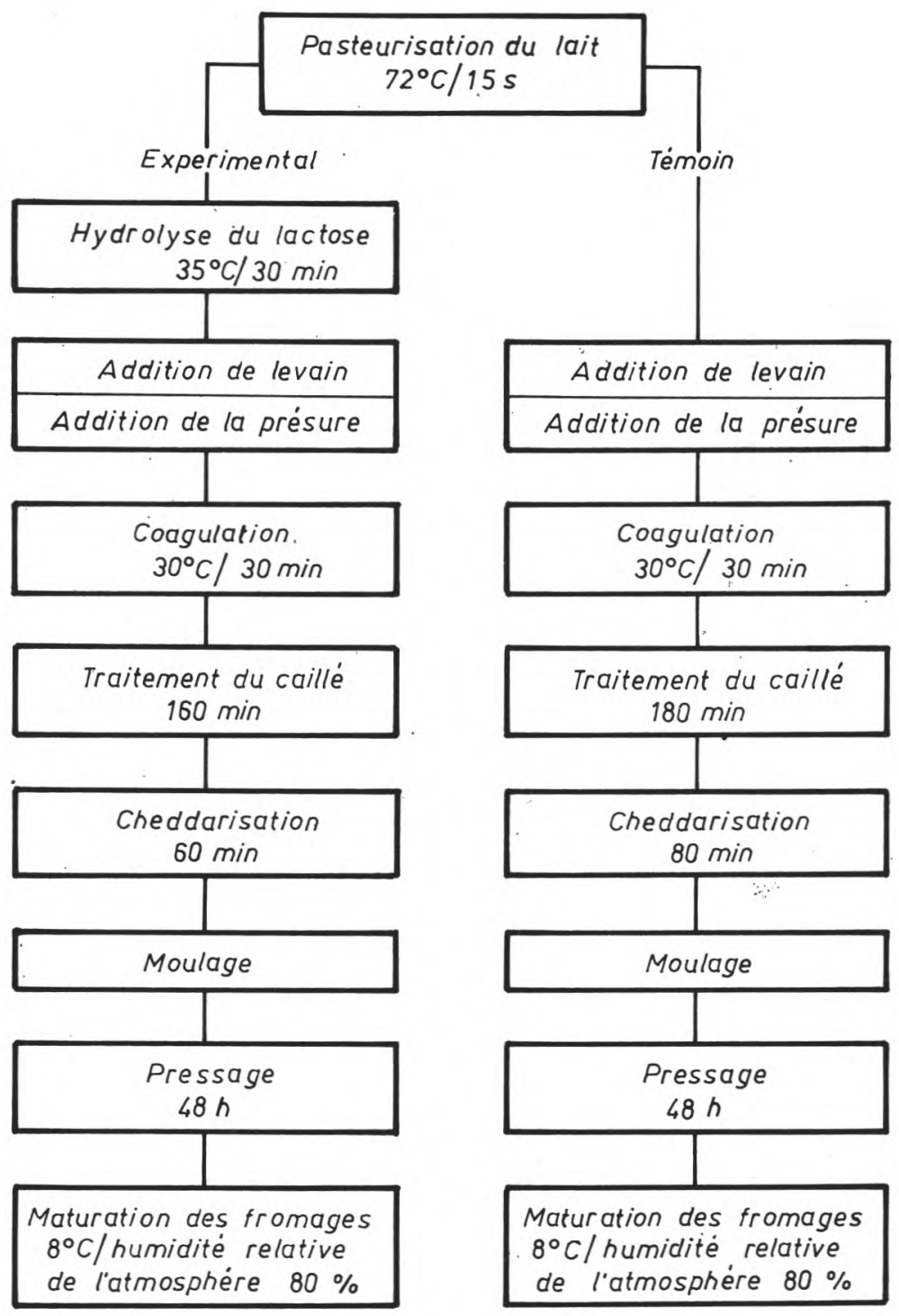

Diagramme schématique de la technologia du Cheddar. 


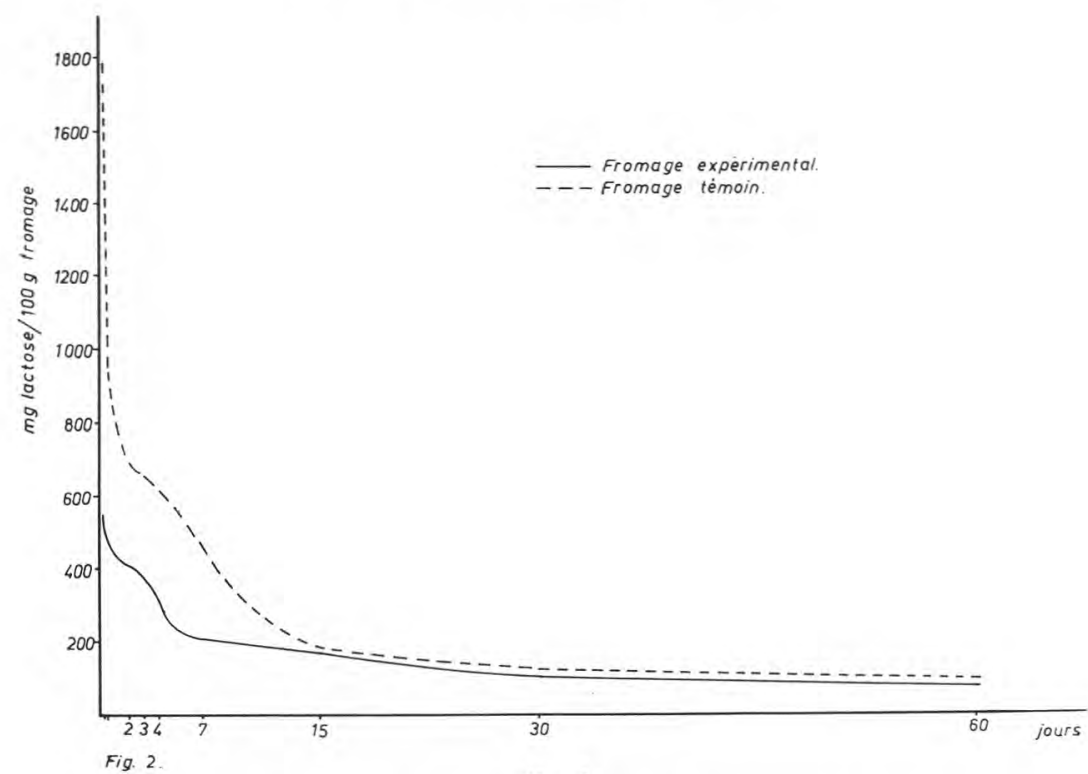

fig. 2

Evolution de la teneur en lactose au cours de la maturation des fromages.

\section{RESULTATS}

L'analyse chromatographique des sucres dans les fromages des fabrications témoin et essai révèle des différences dans les teneurs en lactose, $\gamma$ - $\Delta$-galactose, $\beta$ - $\Delta$-galactose, $\beta$-glucose, surtout au cours de la première étape de la maturation (tab. 1, fig. 1). Les résultats obtenus sur le fromage expérimental (fig. 1b) montrent l'influence de l'hydrolyse enzymatique du lactose dans le lait sur l'intensité des transformations. Par comparaison avec le fromage témoin (fig. 1a) on constate de nettes différences dans la nature et les proportions relatives des sucres analysés. Dans le fromage expérimental on observe la présence de $\gamma$ - $\Delta$-glucose qui n'est pas décelée dans le fromage témoin. Dès le premier stade de maturation, $6 \mathrm{~h}$ après pressage, on note aussi une teneur en lactose plus faible dans le fromage expérimental, 543,2 mg p. 100 contre $1792,6 \mathrm{mg} \mathrm{p} .100$ dans le fromage témoin, et des teneurs en monosaccharides plus élevées : $\gamma$ - $\Delta$-galactose, $416,5 \mathrm{mg}$ p. 100 contre $119,7 \mathrm{mg} \mathrm{p} .100 ; \beta$ - $\Delta$-galactose, 1030,3 mg p. 100 contre $289,3 \mathrm{mg}$ p. $100 ; \beta$ - $\Delta$-glucose, $465,3 \mathrm{mg}$ p. 100 contre $100,3 \mathrm{mg}$ p. 100. Cependant les niveaux de $\gamma$-galactose dans les deux types de fabrications sont peu différents (tab. 1).

La cinétique des transformations du galactose et du glucose est intéressante à observer (fig. 2, 3,4). Dans le fromage expérimental, malgré une teneur en ces sucres plus élevée dans la première étape 


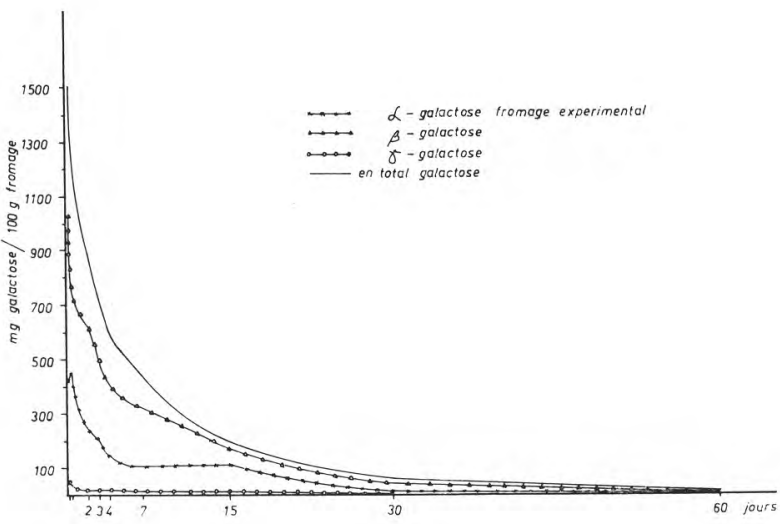

Fig. 30

fig. $3 \mathrm{a}$

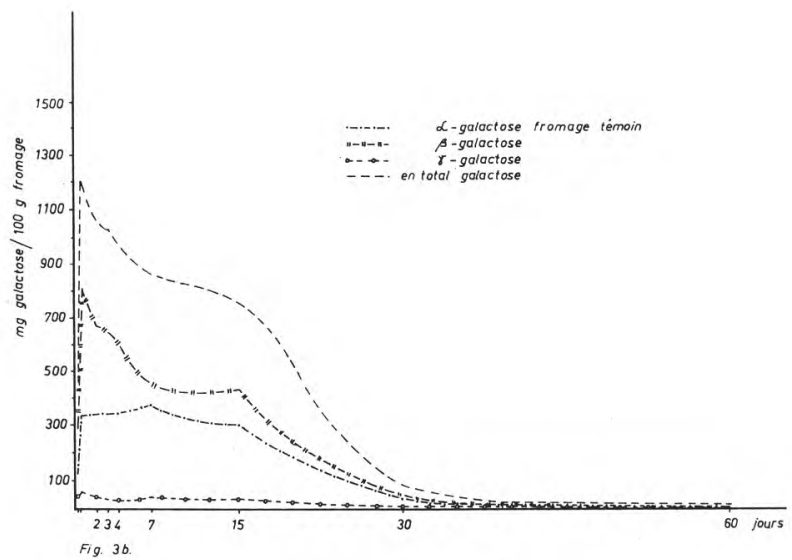

fig. $3 \mathrm{~b}$

fig. 3

Evolution de la teneur en galactose au cours de la maturation des fromages.

a) Fromage expérimental.

b) Fromage témoin.

de la maturation (6 et $12 \mathrm{~h}$ après le pressage), on constate des transformations plus intensives et une fermentation plus rapide que dans le fromage témoin. A titre d'exemple, après $30 \mathrm{j}$ de maturation la teneur en galactose total dans le fromage expérimental était 57,4 mg p. 100 contre $84,5 \mathrm{mg} \mathrm{p.} 100$ dans le fromage témoin (tab. 1, fig. 3). On relève aussi des différences d'intensité dans les transformations du $\gamma$ - $\Delta$-galactose, surtout jusqu'au $15^{\mathrm{e}}$ jour de maturation et du $\beta-\Delta$ galactose (fig. 3). L'utilisation de $\beta$-galactosidase dans la fabrication du Cheddar active aussi les transformations du glucose. Après $30 \mathrm{j}$ 


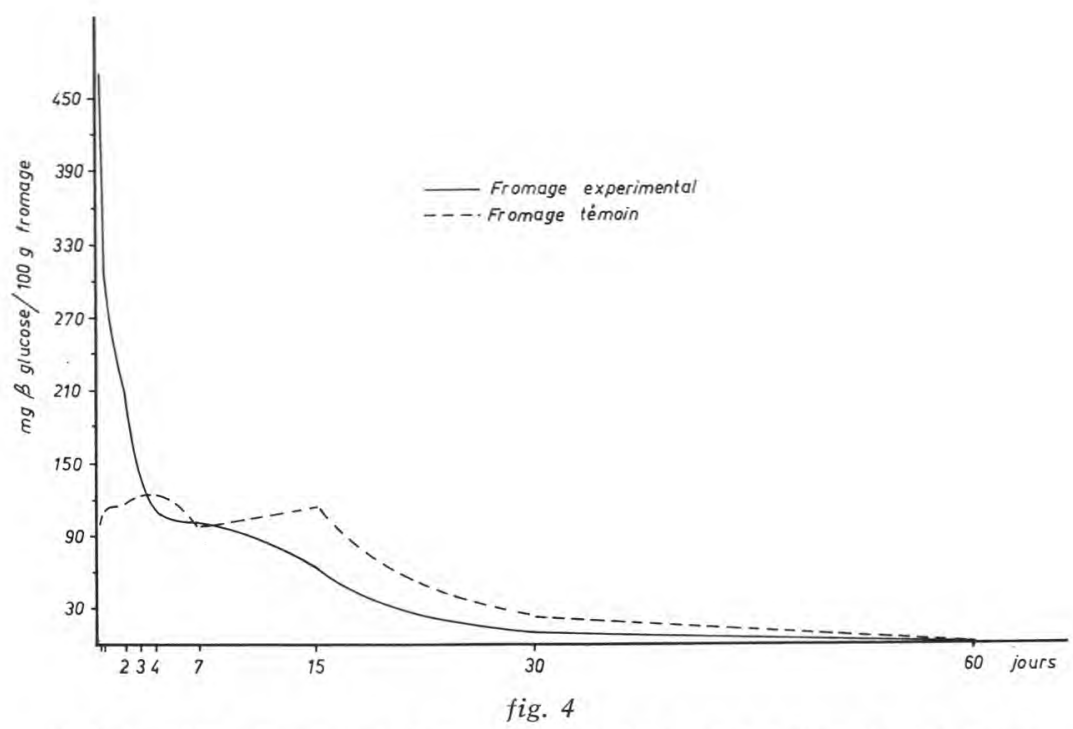

Evolution de la teneur en glucose au cours de la maturation des fromages.

de maturation on trouve dans le fromage expérimental 9,1 mg.p. 100 de glucose contre 22,8 mg p. 100 dans le fromage témoin (tab. 1, fig. 4).

Les différences des teneurs en sucres totaux des deux fabrications diminuent avec le temps de maturation. Après $60 \mathrm{j}$, le fromage expérimental contient $79,7 \mathrm{mg}$ p. 100 de sucres dont 86,5 p. 100 de lactose, le fromage témoin 101,3 mg p. 100 de sucres dont 89,7 p. 100 de lactose (tab. 1).

Le niveau plus élevé des sucres simples, surtout du glucose, dans le lait et dans la masse fromagère, active la multiplication des bactéries introduites avec le levain. Dans le fromage expérimental, $6 \mathrm{~h}$ après pressage, le nombre de bactéries acidifiantes est 100 fois plus grand que dans le fromage témoin $\left(2,78 \times 10^{6} / \mathrm{g}\right.$ contre $\left.2,5 \times 10^{4} / \mathrm{g}\right)$. Un rapport 100/1 est observé pour les nombres de bactéries déterminés sur milieu gélosé à base de lait et de glucose $\left(2,18 \times 10^{9} / \mathrm{g}\right.$ contre $2,15 \times 10^{7} / \mathrm{g}$ ). Des différences dans les populations en bactéries protéolytiques sont également établies, $1,85 \times 10^{6} / \mathrm{g}$ dans le fromage expérimental et $7,1 \times 10^{5} / \mathrm{g}$ dans le fromage témoin (tab. 2).

D'après les données présentées dans le tableau 3 il apparaît que les pourcentages des formes solubles de l'azote sont plus élevés dans les fromages expérimentaux que dans les fromages témoins. Les teneurs en produits de faible poids moléculaire notamment, déterminées comme azote peptidique et azote aminé, montrent que la dégradation des protéines est plus rapide dans les fromages fabriqués avec la préparation de $\beta$-galactosidase. 
TABLEAU 2

Microflore des fromages de Cheddar

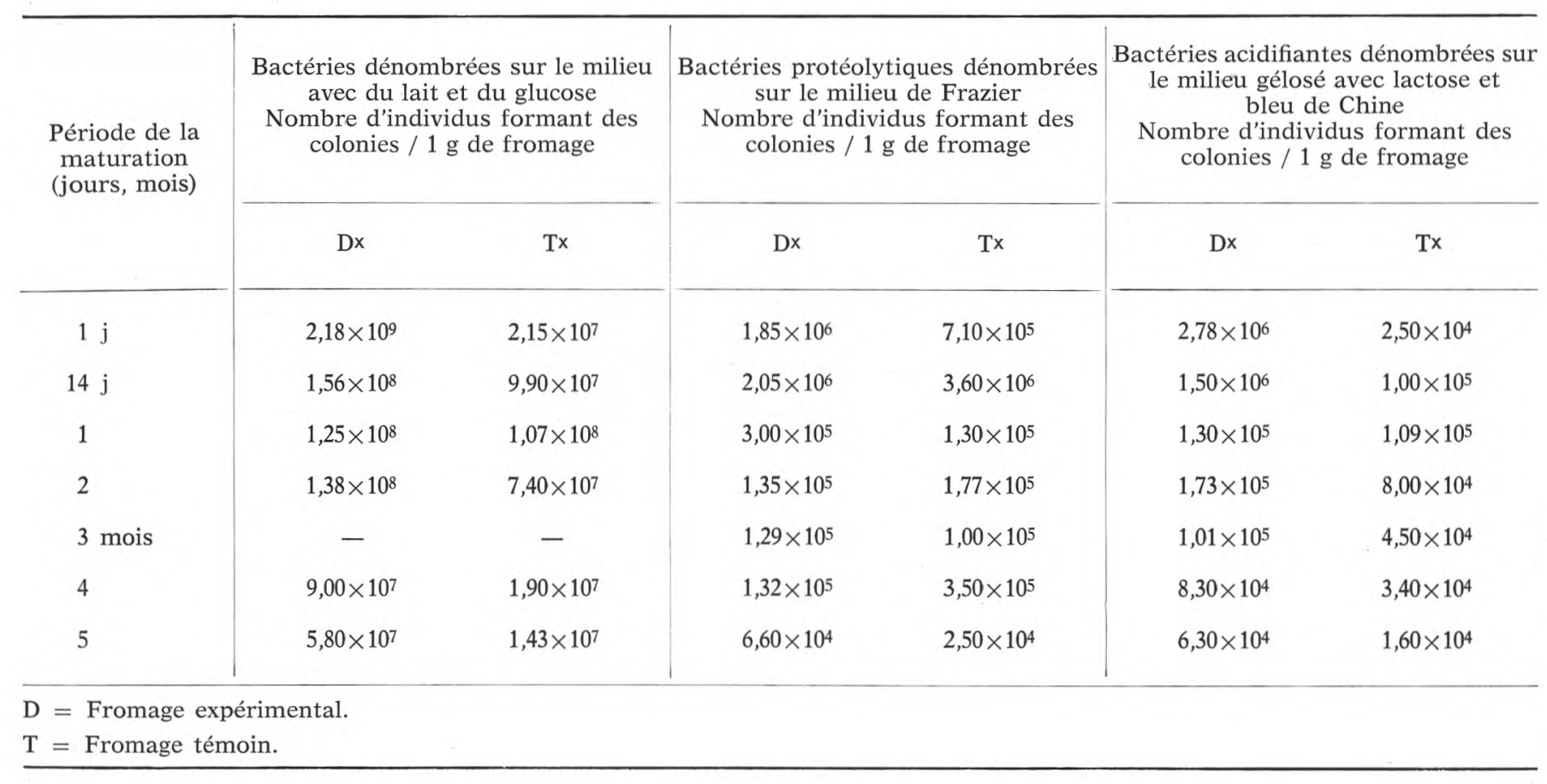


TABLEAU 3

Teneur des matières azotées du Cheddar

\begin{tabular}{|c|c|c|c|c|c|c|c|c|c|c|}
\hline \multirow{3}{*}{$\begin{array}{l}\text { Période de la } \\
\text { maturation } \\
\text { (mois) }\end{array}$} & \multirow{2}{*}{\multicolumn{2}{|c|}{$\begin{array}{l}\text { Azote total } \\
\quad \text { (p. 100) }\end{array}$}} & \multicolumn{8}{|c|}{ Matières azotées en pourcentage d'azote total } \\
\hline & & & \multicolumn{2}{|c|}{$\begin{array}{l}\text { Azote soluble } \\
\text { à pH } 4,6\end{array}$} & \multicolumn{2}{|c|}{$\begin{array}{c}\text { Azote } \\
\text { non protéique }\end{array}$} & \multicolumn{2}{|c|}{ Azote peptidique } & \multicolumn{2}{|c|}{ Azote aminé } \\
\hline & $\mathrm{D}$ & $\mathrm{T}$ & $\mathrm{D}$ & $\mathrm{T}$ & $\mathrm{D}$ & $\mathrm{T}$ & $\mathrm{D}$ & $\mathrm{T}$ & $\mathrm{D}$ & $\mathrm{T}$ \\
\hline 1 & 4,14 & 4,23 & 16,42 & 14,70 & 8,99 & 7,33 & 2,89 & 1,84 & 5,31 & 3,31 \\
\hline 2 & 4,15 & 4,25 & 18,07 & 15,76 & 9,39 & 8,70 & 3,13 & 2,59 & 7,95 & 5,17 \\
\hline 3 & 4,18 & 4,36 & 20,57 & 16,05 & 12,44 & 8,71 & 3,82 & 1,90 & 10,04 & 8,00 \\
\hline 4 & 4,22 & 4,38 & 21,80 & 16,21 & 17,53 & 10,50 & 4,97 & 2,74 & 16,48 & 9,82 \\
\hline 5 & 4,09 & 4,16 & 21,75 & 26,44 & 14,20 & 12,50 & 5,25 & 4,56 & 13,42 & 12,02 \\
\hline \multicolumn{11}{|c|}{$\begin{array}{l}=\text { Fromage expérimental } \\
=\text { Fromage témoin. }\end{array}$} \\
\hline
\end{tabular}




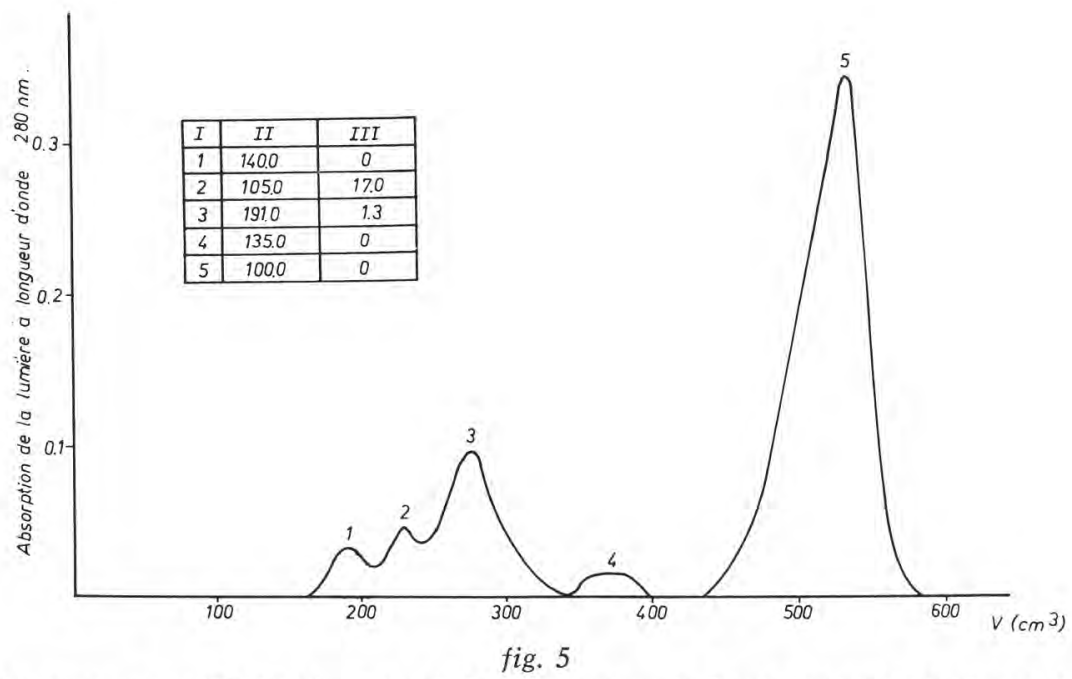

Séparation sur DEAE Séphadex de la préparation de $\beta$-galactosidase Maxilact [6].

I. $\mathrm{N}^{\circ}$ de la fraction.

II. Activité $\beta$-galactosidase exprimée en unités ONPG.

III. Activité protéolytique exprimée en unités d'activité d'après WeSTHOF et al. [20].

Cette hydrolyse plus rapide des protéines des fromages expérimentaux pourrait être partiellement due au fait que la préparation de $\beta$-galactosidase utilisée n'était pas totalement exempte d'enzymes protéolytiques. Le diagramme chromatographique de la préparation " Maxilact 40000 » révèle la présence de 5 fractions dont 2 manifestent une activité protéolytique (fig. 5). La relative faiblesse de cette activité laisse penser que l'intensification de la protéolyse au cours de la maturation a été essentiellement causée par la transformation plus active des sucres; celle-ci a entraîné une stimulation du développement des bactéries dans le lait et dans la masse du fromage et par suite une libération de plus grandes quantités d'enzymes bactériennes provoquant ainsi une protéolyse accrue dans les fabrications expérimentales.

\section{DISCUSSION}

Les résultats obtenus ont montré la possibilité d'intensifier les transformations du lactose et des protéines dans la fabrication du Cheddar. L'utilisation de $\beta$-galactosidase a permis une augmentation des teneurs en monosaccharides, glucose et galactose dans le lait et celle-ci a favorisé un accroissement important du nombre des bactéries responsables de l'acidification au cours de la fabrication du fromage, ainsi que de l'intensité de la maturation. 
Dans la fabrication expérimentale, on a observé une réduction du temps de coagulation du lait de 5 min et du temps de traitement de la masse fromagère de $15 \mathrm{~min}$. On a obtenu un caillé très compact, ce qui a entraîné une diminution de la pulvérisation de la pâte et ainsi une augmentation du rendement. Les produits expérimentaux obtenus avec $1000 \mathrm{l}$ de lait ont retenu $8,7 \mathrm{~kg}$ de composants de matière sèche de plus que les fromages témoins fabriqués en parallèle.

L'influence stimulante de l'hydrolyse du lactose par la $\beta$-galactosidase sur le développement des bactéries dans le lait et, surtout, dans la première étape de la maturation des fromages est confirmée par les travaux de Gilliland et al. [5], de Marschke et al. [8], de Sutherland et al. [18].

Dans le travail présenté, un fait intéressant apparaît avec la possibilité d'utiliser, dans la fabrication des fromages, des préparations de $\beta$-galactosidase moins purifiées, contenant aussi des enzymes protéolytiques utiles pour une intensification de la maturation. Ce point devrait cependant être confirmé par des recherches nouvelles.

L'intensification des transformations des sucres dans la fabrication du Cheddar a une certaine importance économique. On a démontré l'augmentation du rendement et la possibilité de réduire le temps de maturation, d'un mois au minimum, sans influence défavorable sur la qualité des fromages. Un avantage supplémentaire est la récupération d'un lactosérum dont le lactose est hydrolysé et par suite susceptible d'être plus facilement utilisé en boulangerie et pâtisserie car la cristallisation du sucre est alors évitée.

\section{Rés u mé}

Pour intensifier les transformations du lactose dans la fabrication du Cheddar on a utilisé une préparation de $\beta$-galactosidase Maxilact. On a montré la possibilité d'intensifier les transformations des sucres, de réduire le temps de traitement du caillé, d'augmenter le nombre de bactéries acidifiantes et protéolytiques, ainsi que la possibilité d'une protéolyse plus profonde.

Dans les produits expérimentaux on a obtenu un rendement accru de 10 p. 100 et une réduction d'un mois du temps de maturation des fromages.

\section{S u m m a ry}

\section{INTENSIFICATION OF LACTOSE TRANSFORMATION IN CHEDDAR CHEESE PRODUCTION}

Preparate of $\beta$-galactosidase Maxilact was used to intensification of lactose hydrolysis in Cheddar cheese production. The results indicate that is possible to short of time cheese making, to accelerate 
growth of starter bacteria and consequently to increase ripening cheese rate.

The yield of cheese in experimental production was $10 \%$ higher than in control one and ripening time was shorter about one month.

\section{Bibliographie}

[1] Bednakski (W.), Poznanski (S.), Kowalewska (J.), Leman (J.) (1975), Charakterystyka enzymatycznych przemian laktozy w serze, Przeglad Mleczarski, 24 (8), 4-6.

[2] Brittain (G. D.) and Schawe (L.) (1971). - Recent Advances in Gas Chromatography. Domsky J. J., Perry J. A, Editors. Marcel Dekker Pub. Inc., New York.

[3] Burbianka (M.), Pliszka (A.), Janczura (E.), Teisseyre (T.), Zaleska (H.) (1971). - Mikrobiologia Zywnosci. Mikrobiologiczne metody badania produktow zywnosciowych. PZWL Warszawa.

[4] Fagen (J. J.), Stine (J. B.) and Hussong (R. V.) (1952), - The identification of reducing sugars in Cheddar cheese during the early stage of ripening. J. Dairy Sci., 35 (4), 779-781.

[5] Gillilland (E. S.), Speck (M. L.) and Woodard (J. R.) (1972). - Stimulation of lactic Streptococci in milk by $\beta$-galactosidase. Appl. Microbiol., 23, 21-23,

[6] GoodA (E.) (1981). - The charakterization and application of $\beta$-galactosidase preparations. Dysertation. University of Olsztyn.

[7] Köning (P. I. Dc.) (1962), - Peptides from cheese. XVI Int. Dairy Congr., 362.

[8] Marschke (R. J.) and Dulley (J.R.) (1978). - The effect of partial lactose hydrolysis on the manufacture and ripening of Cheddar cheese Austr. J. Dairy Technol., 33, 139-142.

[9] Mc Gugan (W. A.), Emmons (D. D.) and Larmoud (F.) (1979). - Influence of volatile and non volatile fractions on intensity of Cheddar cheese flavour. J. Dairy Sci., 62, 398-399.

[10] Mogensen (M. T.S.) (1948). - Determination of the degree of proteolytic decomposition in cheese with special reference to the formol titration. Moddelande Fran Statens Mejeri-Forsok, 21.

[11] Nilsson (R.) and Guldstrand (M.) (1959). - The separation and quantitative determination of lactose, galactose and glucose in cheese. XV Int. Dairy Congress, 3, 1773.

[12] Pettersson (H. E.) and Sjostrom (G.) (1975). - Accelerated cheese ripening. A methods for increasing the number of lactic starter bacteria in cheese without deterimental effect to the cheese making. J. Dairy Res., 42, 313-326.

[13] PotTeR (F. E.) (1970). - Colorimetric method for the quantitative determination of the degree of lactose hydrolysis. J. Dairy Sci, 53 (2), 803-805.

[14] Readsveld (W.) (1957). - Het verloop de omzetting van lactose tijdens de bereiding van nederland Kaas. Neth. Milk Dairy J., 11, 313-315.

[15] Reiter (B.), Fryer (F.), Chapman (H. R.), LaWrence (R. C.) and Sharp (M. E.) (1967). - The effect of microbiol flora on the flavour and free fatty acid composition of Cheddar cheese. J. Dairy Res., 34, 257-259. 
[16] Schober (K.), Niclaus (W.) und Christ (W.) (1964). - Anwendung der Finger - Abdruck. Method und der Tragen Verdraguugschromatograph auf dic kenuzeichung des reifungsverlanfes von Tilsites, Camembert und Limburger. Käse Milchwiss., 19, 72-73.

[17] Stadhouders (J.) (1960). - The hydrolysis of protein during the ripening of Duch cheese. The enzymes and Bacteria involuid. Neth. Milk Dairy J., $14,106-110$.

[18] Sutherland (B. J.) and Van LeEwin (H. J. M.) (1967). - Estimation of lactose in cheese. Aust. J. Dairy Technol., 22, 221-223.

[19] WebB (B. H.) and Johanson (A. H.) (1965). - Fundamentals of Dairy Chemistry Chap. 6. Avi Publishing Co., Inc. Westport Conn.

[20] Westhoff (D. C.), Cowman (R. A.) and SPeck (M. L.) (1971). - Isolation and partial characterization of particulete proteinase from a slow acid producing mutant of Str. lactis. J. Dairy Sci., 54, 1253-1255. 